\title{
Lymphoma 101: The Plot Thickens
}

\author{
Presented by Leo I. Gordon, MD
}

\section{ABSTRACT}

Staging in lymphoma has changed significantly over time, from the Ann Arbor criteria (1971) to the Cotswolds criteria (1989) to the Lugano Classification (2011). The evolution of imaging technology has played a major role in these changes, and PET/CT is now standard for certain lymphomas, whereas chest radiographs are no longer routine. More recently, response criteria have been refined to account for possible flare reactions of immunomodulatory therapy with a provisional term called "indeterminate response." The latest RECIL criteria use single-dimension measurements to assess response to therapy in patients with lymphoma. Finally, clinical data can be represented graphically in many ways, including the Kaplan-Meir plot, forest plot, waterfall plot, and swimmers plot. Each representation has its own strengths and limitations.

J Natl Compr Canc Netw 2020;18(12.5):1742-1743 doi: $10.6004 /$ jnccn.2020.5031

Driven in part by advances in imaging technology, clinical staging in lymphoma has evolved significantly since the 1970s. At the NCCN 2020 Virtual Congress: Hematologic Malignancies, Leo I. Gordon, MD, Abby \& John Friend Professor of Cancer Research, Professor in Medicine, and Co-Director of the Hematologic Malignancies Program, Robert H. Lurie Comprehensive Cancer Center of Northwestern University, discussed the history of staging using Hodgkin lymphoma as a model, uniform staging criteria for clinical trials, the evolution of response criteria, and graphic representations of clinical trial data.

\section{History of Clinical Staging}

As Dr. Gordon explained, the history of staging in lymphoma dates back to a symposium conducted at the University of Michigan in Ann Arbor in $1971 .{ }^{1}$ The Ann Arbor staging system applied only to the initial presentation of Hodgkin lymphoma and was based on curative treatment with radiation. It was also the first staging criteria to recognize the underlying biology of Hodgkin lymphoma, in that it spreads through contiguity unlike many other malignancies.

These criteria served as the basis of approach to Hodgkin lymphoma until the Cotswolds criteria were introduced in $1989 .{ }^{2}$ CT scans were included, and laparotomy was no longer required. In addition, focal lesions in the liver and spleen were recognized as part of staging, and liver function abnormalities were ignored. The Cotswolds criteria also introduced the " $\mathrm{X}$ " designation for bulky disease and the concept of complete response, unconfirmed $(\mathrm{CRu})$.

\section{CT Versus History and Physical Examination}

According to Dr. Gordon, the history and physical examination have been largely superseded by "somewhat more objective" evaluation with CT imaging, but the benefit to detect recurrence may be the same with both approaches. At the same time, the cost of a history and physical is significantly lower $(\$ 10,000$ per scan vs $\$ 250$ per level 4 visit) and radiation exposure is avoided.

"For staging of almost any malignancy, it is not necessary to do a CT of the chest, abdomen, and pelvis with and without contrast," Dr. Gordon emphasized. "It should be done either with contrast or without contrast, because the radiation exposure doubles."

\section{Response Criteria in Lymphoma Clinical Trials}

The Lugano classification, which was introduced in 2011, had several overarching goals: to improve evaluation of patients with lymphoma, eliminate ambiguity, be universally applicable, facilitate comparison of patients and results among studies, and simplify the evaluation of new therapies by regulatory agencies. ${ }^{3}$ In the Lugano criteria, PET/CT is standard for fluorodeoxyglucose (FDG)-avid lymphomas and CT is standard for nonavid histologies.

Based on the modified Ann Arbor staging, however, patients are treated according to prognostic risk factors for Hodgkin lymphoma, said Dr. Gordon. In addition, A and B classifications only apply to Hodgkin lymphoma, and not to diffuse large B-cell lymphoma, and the X designation was eliminated for bulky disease. Finally, bone marrow aspirate and biopsy were no longer required for large cell lymphoma and Hodgkin lymphoma if the PET scan showed no uptake in marrow. ${ }^{3}$

The Lugano classification was further refined to include treatment with immunomodulatory therapy, the so called LYRIC criteria. ${ }^{4}$ As Dr. Gordon explained, some patients treated with checkpoint inhibitors and other immunotherapies experience a flare reaction. A provisional term, indeterminate response (IR), was introduced to identify lesions that may be a flare reaction versus progressive disease and consists of the following:

- IR1: $\geq 50 \%$ increase in overall tumor burden, determined as the sum of the product of the perpendicular 
diameters of up to 6 lesions in first 12 months of therapy but with no concomitant deterioration in performance status or other signs of progressive disease;

- IR2: New lesions or $\geq 50 \%$ increase of $\geq 1$ existing lesions at any time during treatment, but not overall progression but with no concomitant deterioration in performance status or other signs of progressive disease; and

- IR3: Increase in FDG in $\geq 1$ lesions with no concomitant increase in lesion size or number and with no concomitant deterioration in performance status or other signs of progressive disease.

"The message here is to recognize the potential for flare reactions and to not reassess too early," said Dr. Gordon. "If you have a reassessment that's built in a protocol, you have to recognize the flare reaction and evaluate imaging response in concert with a clinical response. The idea is to prevent prematurely stopping a therapy that may be effective."

In the future, said Dr. Gordon, measuring the change in standardized uptake value, total tumor glycolysis, and metabolic tumor volume may provide quantitative assessment of response.

\section{RECIL Response Criteria}

As Dr. Gordon explained, the Lugano criteria are based on FDG-PET or bidimensional tumor measurements on CT scan. These differ from RECIST criteria used in solid tumors, which use unidimensional measurements. The RECIL group hypothesized that single-dimension measurements could be used to assess response to therapy in patients with lymphoma, producing results similar to the standard criteria. By analyzing imaging measurements from 10 multicenter clinical trials, the group developed new lymphoma response criteria (RECIL 2017). ${ }^{5}$

According to Dr. Gordon, the RECIL group showed that assessment of tumor burden in lymphoma clinical trials can use the sum of the longest diameters of a maximum of 3 target lesions, in contrast with RECIST and Lugano criteria, which both require up to 6 target lesions. The RECIL criteria also introduced a new provisional category of minor response, "which may be important," Dr. Gordon said. Furthermore, the RECIL criteria clarified response assessment in patients receiving novel immune therapy and targeted agents that generate unique imaging situations.
"These criteria still have to be worked out and haven't been used quite as much in clinical trials as the other criteria," said Dr. Gordon, "but I think it's important to be aware of this."

\section{Graphical Representation of Clinical Trial Data}

The most common plot used by statisticians is the Kaplan-Meier plot, which is an estimate of survival over time. Based on an article by Kaplan and Meier, the plot provides a snapshot of survival at various time intervals. ${ }^{6}$

"You can pick any time point, look at the 2 curves, and compare the survival probability or progression-free survival probability at any time point between 2 treatment arms," Dr. Gordon explained. "This is very useful and important, but it is still an estimation from incomplete observations."

The forest plot is another way to graphically display data, and is useful when trying to analyze subgroups. One caveat, however, is that statistical power to make decisions is reduced as the numbers in a group decrease, said Dr. Gordon.

The waterfall plot is a granular way of evaluating response to treatment and provides a very quick, graphic interpretation of a clinical trial, whereas the swimmers plot gives an idea about treatment duration and the length of response to treatment. These plots offer a statistical estimate of outcomes, but may ignore what relapse means to an individual patient. Dr. Gordon proposed a new way of thinking about relapses in diseases such as follicular lymphoma, in which there is controversy about maintenance treatments that improve progression-free survival but not overall survival. The impact of recurrence (which may be more frequent in the absence of maintenance therapy) on the life of a patient and their family might be designated as a "lightning bolt" for the patient, and therefore the "lightening plot" is Dr. Gordon's way of reminding oncologists that decisions should not be based solely on data points on a curve, but must also take into consideration patients' wishes and the impact of those decisions on patients' lives. However, this is not an accepted measure in clinical trials yet.

Disclosures: Dr. Gordon has disclosed that he has received grant/research support from Celgene Corporation and Juno Therapeutics, Inc., is a scientific advisor for Gilead Sciences, Inc.; and has an ownership/partnership/principal role with Zylem Biosciences.

Correspondence: Leo I. Gordon, MD, Northwestern University, 676 North St Clair, Suite 850, Chicago, IL 60611. Email: I-gordon@northwestern.edu

\section{References}

1. Carbone PP, Kaplan HS, Musshoff K, et al. Report of the Committee on Hodgkin's Disease Staging Classification. Cancer Res 1971;31:1860-1861.

2. Lister TA, Crowther D, Sutcliffe SB, et al. Report of a committee convened to discuss the evaluation and staging of patients with Hodgkin's disease: Cotswolds meeting. J Clin Oncol 1989;7:1630-1636.

3. Cheson BD, Fisher RI, Barrington SF, et al. Recommendations for initial evaluation, staging, and response assessment of Hodgkin and non-Hodgkin lymphoma: the Lugano classification. J Clin Oncol 2014;32:3059-3068.

4. Cheson BD, Ansell S, Schwartz L, et al. Refinement of the Lugano Classification lymphoma response criteria in the era of immunomodulatory therapy. Blood 2016;128:2489-2496.

5. Younes A, Hilden P, Coiffier B, et al. International Working Group consensus response evaluation criteria in lymphoma (RECIL 2017). Ann Oncol 2017; 28:1436-1447.

6. Kaplan EL, Meier P. Nonparametric estimation from incomplete observations. J Am Stat Assoc 1958;53:457-481. 